\title{
COMMENTARY
}

\section{Moving beyond the 'pancreatic rest' in severe and critical acute pancreatitis}

\author{
Maxim S Petrov* \\ See related research by Chang et al., http://ccforum.com/content/17/3/R118
}

\begin{abstract}
Nasojejunal tube feeding is considered the current standard of care in patients with severe and critical acute pancreatitis. However, it is not known whether enteral nutrition is best delivered into the jejunum. This Commentary discusses recent clinical studies that have shown that tube feeding into the stomach is safe and well tolerated in the vast majority of patients with acute pancreatitis, thus overthrowing the notion of putting the pancreas at rest. Development of a new conceptual framework is warranted to further advance nutritional management of patients with acute pancreatitis.
\end{abstract}

Enteral nutrition is a rapidly evolving frontier in the management of acute pancreatitis (AP). In the previous issue of Critical Care, Chang and colleagues investigate whether nasojejunal tube feeding confers any tangible benefit compared with nasogastric tube feeding in patients with AP [1]. It has been 5 years since publication of the previous systematic review on the topic [2] and it is timely to review the progress. Further, the recent international multidisciplinary classification of AP has redefined the 'severe' category of severity and introduced the new 'critical' category of severity (Table 1), thus putting a high emphasis on the need to optimise management of these most challenging patients [3-6].

The study by Chang and colleagues [1] adds an important perspective to the discussion regarding the 'pancreatic rest' concept, which is perhaps the oldest dogma in the management of AP. The central tenet of this concept is that enteral nutrition delivered into any part of the upper gastrointestinal tract other than the jejunum stimulates pancreatic secretion and, consequently, exacerbates the severity of AP. The corollary is that

\footnotetext{
*Correspondence: max.petrov@gmail.com
}

Department of Surgery, The University of Auckland, Auckland 1142, New Zealand 'non-stimulatory' nutrition had been widely advocated, being total parenteral nutrition two to three decades ago and nasojejunal tube feeding in the past decade. That is why the majority of randomised controlled trials in the past studied 'non-stimulatory' regimens as both intervention and comparator, that is, either parenteral nutrition versus nil per os, or parenteral nutrition versus jejunal tube feeding, or jejunal tube feeding versus nil per os $[7,8]$. It is argued that this has retarded progress in the field.

The systematic literature review by Chang and colleagues [1] has appraised the current best evidence regarding the use of nasogastric tube feeding (presumed to be 'stimulatory') in patients with AP. It demonstrates that the evidence base is (still) relatively small but does show that enteral nutrition given via the nasogastric route is well tolerated in more than $90 \%$ of patients with AP [9-11]. In line with the previous systematic review [2], it shows no statistically significant difference between 'non-stimulatory' and 'stimulatory' regimens in terms of morbidity and mortality. The new, and somewhat surprising, finding here is that both routes of enteral feeding appear to be equivalent in terms of delivery of target calories.

There are two possible explanations for the observed results. First, tube feeding into the stomach might have been 'non-stimulatory' in patients with AP. Unfortunately, little is known about the secretory response of the pancreas during the acute phase of clinical AP, let alone the effect of feeding on it [12]. But a study in healthy volunteers demonstrated that both oral and duodenal tube feeding stimulate pancreatic enzyme secretion in comparison with placebo [13]. Moreover, the degree of pancreatic stimulation is very similar between oral and duodenal tube feeding. Second, tube feeding into the stomach might have stimulated the pancreas in patients with AP but it has no clinical ramifications, essentially meaning that the concept of 'pancreatic rest' might have been fallacious. Although it has become deeply entrenched in the management of AP, it is worth noting that the 'pancreatic rest' concept was never proven in randomised controlled trials. Moreover, the recent MIMOSA (MIld to MOderate acute pancreatitis: early 
Table 1. Definitions of the four severity categories according to the $\mathbf{2 0 1 2}$ international multidisciplinary classification of acute pancreatitis [4]

\begin{tabular}{lcccc}
\hline & Mild & Moderate & Severe & Critical \\
\hline (Peri)pancreatic necrosis & No & Sterile & Infected & Infected \\
& AND & AND/OR & OR & AND \\
Organ failure & No & Transient & Persistent & Persistent
\end{tabular}

(Peri)pancreatic necrosis is nonviable tissue located in the pancreas alone, or in the pancreas and peripancreatic tissues, or in peripancreatic tissues alone. It can be solid or semisolid (partially liquefied) and is without a radiologically defined wall. Sterile (peri)pancreatic necrosis is the absence of proven infection in necrosis. Infected (peri)pancreatic necrosis is defined when at least one of the following is present: gas bubbles within (peri)pancreatic necrosis on computed tomography; a positive culture of (peri)pancreatic necrosis obtained by image guided fine-needle aspiration; a positive culture of (peri)pancreatic necrosis obtained during the first drainage and/or necrosectomy. Organ failure is defined for three organ systems (cardiovascular, renal, and respiratory) on the basis of the worst measurement over a 24-hour period. In patients without pre-existing organ dysfunction, organ failure is defined as either a score of 2 or more in the assessed organ system using the SOFA (Sepsis-related Organ Failure Assessment) score or when the relevant threshold is breached, as shown: Cardiovascular, need for inotropic agent; Renal, creatinine $\geq 171 \mu \mathrm{mol} / \mathrm{L}$ $\left(\geq 2.0 \mathrm{mg} / \mathrm{dl}\right.$ ); Respiratory, $\mathrm{PaO}_{2} / \mathrm{FiO}_{2}$ (partial pressure of oxygen/fractional inspired oxygen concentration) $\leq 300 \mathrm{mmHg}$ ( $\leq 40 \mathrm{kPa}$ ). Persistent organ failure is the evidence of organ failure in the same organ system for 48 hours or more. Transient organ failure is the evidence of organ failure in the same organ system for less than 48 hours.

naSogastric tube feeding compared with pAncreatic rest) trial compared in a randomized fashion early nasogastric tube feeding (commenced within 24 hours after hospital admission) with nil per os and found that nasogasric feeding does not exacerbate the course of AP and even reduces the risk of oral food intolerance [14]. Similarly, an earlier randomised controlled trial compared early nasogastric tube feeding (commenced within 24 hours after hospital admission) with parenteral nutrition and found no difference between 'non-stimulatory' and 'stimulatory' regimens [15].

In conclusion, accumulating evidence indicates that the site of enteral tube feeding does not affect major clinical outcomes in patients with AP. Given that tube feeding into the stomach is more practical than into the jejunum in the majority of clinical settings, it should be considered as the first-line approach for patients with severe and critical AP. The 'pancreatic rest' concept can now be put to rest. There is a need and justification for developing a contemporary conceptual framework concerning nutritional management of AP.

\section{Abbreviations}

AP, acute pancreatitis.

\section{Competing interests}

The author declares that he has no competing interests.
Published: 1 July 2013

References

1. Chang Y, Fu H, XiaoY, Liu J: Nasogastric or nasojejunal feeding in predicted severe acute pancreatitis: a meta-analysis. Crit Care 2013, 17:R118.

2. Petrov MS, Correia MI, Windsor JA: Nasogastric tube feeding in predicted severe acute pancreatitis. A systematic review of the literature to determine safety and tolerance. JOP 2008, 9:440-448.

3. Petrov MS, Windsor JA: Conceptual framework for classifying the severity of acute pancreatitis. Clin Res Hepatol Gastroenterol 2012, 36:341-344.

4. Dellinger EP, Forsmark CE, Layer P, Lévy P, Maraví-Poma E, Petrov MS, Shimosegawa T, Siriwardena AK, Uomo G, Whitcomb DC, Windsor JA, Pancreatitis Across Nations Clinical Research and Education Alliance (PANCREA): Determinant-based classification of acute pancreatitis severity: An international multidisciplinary consultation. Ann Surg 2012, 256:875-880.

5. Petrov MS, Windsor JA, Lévy P; Pancreatitis Across Nations Clinical Research and Education Alliance (PANCREA): New international classification of acute pancreatitis: more than just 4 categories of severity. Pancreas 2013, 42:389-391.

6. Maraví Poma E, Zubia Olascoaga F, Petrov MS, Navarro Soto S, Laplaza Santos C, Morales Alava F, Darnell Martin A, Gorraiz López B, Bolado Concejo F, Casi Villarroya M, Aizcorbe Garralda M, Albeniz Arbizu E, Sánchez-Izquierdo Riera JA, Tirapu León JP, Bordejé Laguna L, López Camps V, Marcos Neira P, Regidor Sanz E, Jiménez Mendioroz F: SEMICYUC 2012. Recommendations for intensive care management of acute pancreatitis. Med Intensiva 2013, 37:163-179.

7. Petrov MS, Pylypchuk RD, Emelyanov NV: Systematic review: nutritional support in acute pancreatitis. Alim Pharmacol Ther 2008, 28:704-712.

8. Petrov MS, Loveday BP, Pylypchuk RD, Mcllroy K, Phillips AR, Windsor JA: Systematic review and meta-analysis of enteral nutrition formulations in acute pancreatitis. Br J Surg 2009, 96:1243-1252

9. Eatock FC, Chong P, Menezes N, Murray L, McKay CJ, Carter CR, Imrie CW: A randomized study of early nasogastric versus nasojejunal feeding in severe acute pancreatitis. Am J Gastroenterol 2005, 100:432-439.

10. Kumar A, Singh N, Prakash S, Saraya A, Joshi YK: Early enteral nutrition in severe acute pancreatitis: a prospective randomized controlled trial comparing nasojejunal and nasogastric routes. J Clin Gastroenterol 2006, 40:431-434.

11. Singh N, Sharma B, Sharma M, Sachdev V, Bhardwaj P, Mani K, Joshi YK, Saraya $A$ : Evaluation of early enteral feeding through nasogastric and nasojejunal tube in severe acute pancreatitis: a noninferiority randomized controlled trial. Pancreas 2012, 41:153-159.

12. Dominguez-Munoz JE, Pieramico O, Buchler M, Malfertheiner P: Exocrine pancreatic function in the early phase of human acute pancreatitis. Scand J Gastroenterol. 1995, 30:186-191.

13. O'Keefe SJ, Lee RB, Anderson FP, Gennings C, Abou-Assi S, Clore J, Heuman D, Chey W: Physiological effects of enteral and parenteral feeding on pancreaticobiliary secretion in humans. Am J Physiol Gastrointest Liver Physiol 2003, 284:G27-G36.

14. Petrov MS, Mcllroy K, Grayson L, Phillips AR, Windsor JA: Early nasogastric tube feeding versus nil per os in mild to moderate acute pancreatitis: A randomized controlled trial. Clin Nutr 2012 [Epub ahead of print]

15. Eckerwall GE, Axelsson JB, Andersson RG: Early nasogastric feeding in predicted severe acute pancreatitis: A clinical, randomized study. Ann Surg 2006, 244:959-965.

doi:10.1186/cc12770

Cite this article as: Petrov MS: Moving beyond the 'pancreatic rest' in severe and critical acute pancreatitis. Critical Care 2013, 17:161. 